\begin{tabular}{|c|c|c|c|c|c|c|c|c|c|c|c|c|}
\hline & \multicolumn{3}{|c|}{ CuIm in ation. } & \multicolumn{3}{|c|}{ Grade Aufsteigung. } & \multicolumn{3}{|c|}{ Abweichung. } & \multicolumn{2}{|c|}{ Parallaxe. } \\
\hline & & Log a & 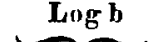 & Log $c$ & og a & & I. & Log a & & Lng c & Lo & $T$ \\
\hline ct & 16 & 2,487348 & 38 & & 3 & 9,33690 & 8,1124 & 3,322132 & $0,02608 n$ & 9,176 & 2,080 & 8,001 \\
\hline & 17 & 97 & 9 & 8,1 & & 9 & 8,1 & & $0,72763 n$ & & & 8,539 \\
\hline & 18 & 953 & 0 & 10 & 33 & 40 & & & & & & \\
\hline & 15 & 621 & 6 & 9 & 830 & 19 & 7 & & & & & -5 \\
\hline & 20 & 757 & 4 & $3 n$ & 066 & 0 & $13 n$ & 12 & & & & 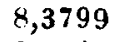 \\
\hline & 2 & 24 & & $7 n$ & 733 & 0 & $3 n$ & 82 & & & & -1 \\
\hline & 2 & 74 & & $n$ & 773 & 9 & $6 n$ & 69 & & & & 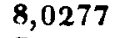 \\
\hline & 2 & 91 & & $3 n$ & 17 & 4 & $29 n$ & $33 n$ & & & & - \\
\hline & 2 & 53 & $4 n$ & $7 n$ & 83 & $3 n$ & $E_{n}$ & $6 n$ & $\ln n$ & & & 7,9027 \\
\hline & 2 & 75 & & $3 n$ & 41 & & $01 n$ & $96 n$ & $6 n$ & & & \\
\hline & 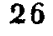 & 7 & & 7 & 43 & $6 n$ & 7,3602 & $54 n$ & $7 n$ & & & \\
\hline & 2 & & & 2 & & & 7,6 & $9 n$ & & & & \\
\hline & 2 & & & 33 & & $6 n$ & 8 & $1 n$ & $0 n$ & & 98 & \\
\hline & 29 & 1 & & & & 3 & 8,1 & $01 n$ & $91 n$ & & 84 & 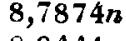 \\
\hline & 30 & 3 & & & & & 8,0 & $6 n$ & & & 89 & 4 \\
\hline & 31 & 60 & & & 1 & & 7,6 & $4 n$ & & & 50.2 & 9 , \\
\hline & $\mathbf{1}$ & 2,02 & 9,98134 & 19. & 608951 & 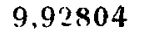 & $7,8024 n$ & $3,266776 n$ & 131 & 9,4940 & $52 n$ & 8,969 \\
\hline & 3 & & & & & & & & & & & \\
\hline & 4 & & & & & & & & & & & \\
\hline & 5 & & & & & & & & 85 & & & \\
\hline
\end{tabular}

Th. Clausen.

\title{
Beitrag zur Theorie der dreiseitigen Pyramide.
} Von Herrn Thomas Clausen.

1. Die Aufgabe: „aus den drei Winkeln zwischen den Kanten an dem Scheitel $A$ einer dreiseitigen Pyramide $A B C D$; nemlich $B A C=A, C A D=\theta^{\prime}$ und $D A B=\theta^{\prime \prime}$; und den drei Kanten der Grundflache $B C=a, C D=a^{\prime}$ und $D B=a^{\prime \prime}$ die übrigen Stücke zu finden;" scheint noch keine einfache Auflösung gefunden zu haben. Monge in seiner Geometrie descriptive sagt, p. 126, dafs die analytische Entwickelung auf eine Gleichung vom achten Grade führe. Die Aufgabe läfst sich aber leicht auf eine Gleichung vom vierten Grade bringen. Es seien nemlich die drei Kanten $A D=r, A B=r^{\prime}$ und $A C=r^{\prime \prime}$; so hat man zwischen diesen und den gegebenen Grörsen folgende Relationen:

$$
\left.\begin{array}{l}
a^{2}=r^{\prime 2}+r^{\prime \prime 2}-2 r^{\prime} r^{\prime \prime} \cos \theta \\
a^{\prime 2}=r^{\prime \prime 2}+r^{2}-2 r^{\prime \prime} r \cos \theta^{\prime}
\end{array}\right\} \ldots \ldots \ldots(\mathrm{I})
$$

Aus ihnen folgt unmittelbar:

$$
\left.\begin{array}{l}
a^{2} r^{2}-a^{\prime 2} r^{\prime 2}+\left(a^{2}-a^{\prime 2}\right) r^{\prime \prime 2}-2 a^{2} r^{\prime \prime} r \cos \theta^{\prime}+2 a^{\prime 2} r^{\prime} r^{\prime \prime} \cos \theta=0 \\
a^{\prime 2} r^{\prime 2}-a^{\prime \prime 2} r^{\prime \prime 2}+\left(a^{\prime 2}-a^{\prime \prime 2}\right) r^{2}-2 a^{\prime 2} r^{\prime \prime} \cos \theta^{\prime \prime}+2 a^{\prime \prime 2} r^{\prime \prime} r \cos \theta^{\prime}=0
\end{array}\right\}
$$

Setzt man $r=\nu r^{\prime \prime}: r^{\prime}=\nu^{\prime} r^{\prime \prime}$, so erhält man:

$$
\left.\begin{array}{l}
a^{2} \nu^{2}-a^{\prime} \nu^{\prime 2}+a^{2}-a^{\prime 2}-2 a^{2} \nu \cos \theta^{\prime}+2 a^{\prime 2} \nu^{\prime} \cos \theta=0 \\
a^{\prime 2} \nu^{\prime 2}-a^{\prime \prime 2}+\left(a^{\prime 2}-a^{\prime \prime 2}\right) \nu^{2}-2 a^{\prime 2} \nu \nu^{\prime} \cos \theta^{\prime \prime}+2 a^{\prime 2} \nu \cos \theta^{\prime}=0 \\
u^{\prime \prime 2}-a^{2} \nu^{2}+\left(a^{\prime \prime 2}-a^{2}\right) \nu^{\prime 2}-2 a^{\prime 2} \nu^{\prime} \cos \theta+2 a^{2} \nu \nu^{\prime} \cos \theta^{\prime \prime}=0
\end{array}\right\}
$$

Diese drei Gleichungen enthalten nur zwei verschiedene, da jede ders̀elben sich aus den beiden ïbrigen ableiten lärst. Betrachtet man $v$ und $v^{\prime}$ als die Coordinaten einer krummen Linie; so können diese Gleichungen in Kegelschnitte entworfen werden, die nicht mehr als vier gemeinschaftliche Puncte haben. Es sind demnach nicht mehr als vier verschiedene Auflösungen möglich, von denen übrigens die, in denen $\nu=\frac{r}{r^{\prime \prime}}$, und $\nu^{\prime}=\frac{r^{\prime}}{r^{\mu}}$ nicht beide positiv sind, ausgeschie- den werden müssen, da alle drei $r, r^{\prime}, r^{\prime \prime}$ immer positiv sind. Aus jedem Paar Werthe von $\nu$ und $v^{\prime}$ folgt wieder nur ein Werth der drei Grölsen $r, r^{\prime}, r^{\prime \prime}$; nemlich

$$
\begin{aligned}
& r^{\prime \prime}=\frac{a}{\sqrt{\left(1-2 \nu^{\prime} \cos \theta+\nu^{\prime 2}\right)}}=\frac{a^{\prime}}{\sqrt{\left(1-2 \nu \cos \theta^{\prime}+\nu^{2}\right)}} \text {; } \\
& r=v r^{\prime \prime} ; r^{\prime}=v^{\prime} r^{\prime \prime} \text {. }
\end{aligned}
$$

da der negative Werth ausgeschlossen ist. Es sind also in allen Fällen nicht mehr als vier Auflösungen der Aufgabe möglich. Alle vier können aber in vielen Fällen möglich sein, 26 * 
z. B. wenn $\cos \theta=\cos \theta^{\prime}=\cos \theta^{\prime \prime}=\frac{2}{3} ; a=a^{\prime}=a^{\prime \prime}=1$ ist; so wird den Gleichungen 1 durch folgende vier Systeme Genüge geleistet:

$$
\begin{aligned}
& \text { 1. } r=\sqrt{\frac{3}{2}} ; \quad r^{\prime}=V_{\frac{3}{2}} ; \quad r^{\prime \prime}=V_{\frac{3}{2}}^{3} \\
& \text { 2. }=\sqrt{\frac{3}{2}} ; \quad=\sqrt{\frac{3}{2}} ;=\sqrt{\frac{1}{6}} \\
& \text { 3. }=\sqrt{\frac{3}{2}} ; \quad=\sqrt{\frac{1}{6}} ; \quad=\sqrt{\frac{3}{2}} \\
& \text { 4. }=\sqrt{\frac{1}{6}} ;=\sqrt{\frac{3}{2}} ;=\sqrt{\frac{3}{2}}
\end{aligned}
$$

2. Man kann die Aufgabe noch auf einem ganz andern Wege auflösen, wobei man blos auf eine cubische Gleichung kümmt, welches im Grunde nicht einfacher ist, als die Auflösung der aus den Gleichungen IlI folgende biquadratische, die man durch Hülfe eiuer cubischen Gleichung in zwei quadra tische zerlegen kann.

Die in der Pyramide eingeschriebene Kugel berübre die vier Seitenflächen $B A C, C A D, D A B, B C D$ resp. in den Puncten $p, p^{\prime}, p^{\prime \prime}, p^{\prime \prime}$. Verbindet man diest vier Puncte zu zweien, durch 6 grö?ste Kreise; so wird in dem sphärischen Dreiecke $p p^{\prime} p^{\prime \prime}$ der Winkel $<p^{\prime \prime} p p^{\prime}=180^{\circ}-\theta ;<p p^{\prime} p^{\prime \prime}$ $=180^{\circ}-\theta^{\prime} ;\left\langle p^{\prime} p^{\prime \prime} p=180^{\circ}-\theta^{\prime \prime}\right.$. Es seien die drei sphärischen Seiten $p^{\prime} p^{\prime \prime}=l, p^{\prime \prime} p=l^{\prime} ; p p^{\prime}=l^{\prime \prime}$. Sei ferner in dem ebenen Dreiecke $\angle C D B=\alpha ; \angle D B C=\alpha^{\prime} ; \quad \angle B C D$ $=\alpha^{\prime \prime}$, oder wenn man

$$
\frac{\sin \alpha}{a}=\frac{\sin \alpha^{\prime}}{a^{\prime}}=\frac{\sin \alpha^{\prime \prime}}{a^{\prime \prime}}=\frac{V\left\{\left(a+a^{\prime}+a^{\prime \prime}\right)\left(a+a^{\prime}-a^{\prime \prime}\right)\left(a-a^{\prime}+a^{\prime \prime}\right)\left(-a+a^{\prime}+a^{\prime \prime}\right)\right\}}{2 a a^{\prime} a^{\prime \prime}}
$$

setzt, so werden die sphärischen Winkel $\left\langle p^{\prime} p^{\prime \prime \prime} p^{\prime \prime}=180-\alpha\right.$, $\left\langle p p^{\prime \prime} p^{\prime \prime}=180-\alpha^{\prime} ;\left\langle p p^{\prime \prime \prime} p^{\prime}=180-\alpha^{\prime \prime}\right.\right.$. Es sind denınach in dem sphärischen Vierecke $p p^{\prime \prime} p^{\prime} p^{\prime \prime \prime}$ zwei Seiten $p^{\prime} p^{\prime \prime}=l$, $p^{\prime \prime} p=l^{\prime}$ und der eingeschlossene Winkel $180-\theta^{\prime \prime}$, ferner die beiden Winkel, die die Diagonale an dem Durchschnittspunct der beiden übrigen Seiten $p p^{\prime \prime}, p^{\prime} p^{\prime \prime \prime}$ nit der Diagonale $p^{\prime \prime} p^{\prime \prime}$ an diesem Durchnitte bilden $\angle p p^{\prime \prime \prime} p^{\prime \prime}=180-\alpha^{\prime} ; \quad<p^{\prime} p^{\prime \prime \prime} p^{\prime \prime}$ $=180-\propto$ gegeben; man soll die übrigen Stücke 'des sphärischen Vierecks bestimmen.

Es sei $p^{\prime \prime} p^{\prime \prime \prime}=y$; die Winkel $<p^{\prime} p^{\prime \prime} p^{\prime \prime \prime}=90-\frac{1}{2} \theta^{\prime \prime}+\xi$, $<p p^{\prime \prime} p^{\prime \prime \prime}=90-\frac{1}{2} \theta^{\prime \prime}-\xi$; so hat man in den Dreiecken $p p^{\prime \prime} p^{\prime \prime}, p^{\prime} p^{\prime \prime} p^{\prime \prime}$

$$
0=h h \cos 2 H \sin \xi^{2}+2 g h \sin (
$$$$
\text { 列 }
$$$$
+\frac{1}{4}\left(e-e^{\prime}\right)^{2} \sin \theta^{\prime \prime 2}+\frac{1}{2}\left(e^{2}-e^{\prime 2}\right) \sin \theta^{\prime \prime} \sin 2 \xi+\frac{1}{4}\left(e+e^{\prime}\right)^{2} \sin 2 \xi^{2}
$$

und wenn man ferner

$$
\begin{aligned}
& T=2 h h \cos 2 H-2 g g \cos 2 G+\left(e-e^{\prime}\right)^{2} \sin \theta^{\prime \prime 2} \\
& U=h h \cos 2 H+g g \cos 2 G \\
& V=2 g h \sin (G+H)+\left(e^{2}-e^{\prime 2}\right) \sin \theta^{\prime \prime} \\
& S=\left(e+e^{\prime}\right)^{2}
\end{aligned}
$$

setzt,

$$
0=T-2 U \cos 2 \xi+2 V \sin 2 \xi+S \sin 2 \xi^{2} .
$$

Dieser gebe ich folgende identische Form

$\mathrm{0}=T-x+x \cos 2 \xi^{2}+(S+x) \sin 2 \xi^{2}-2 U \cos 2 \xi+2 V \sin 2 \xi$ und bestimme $x$ so, dafs sie sich in

(T)..... $0=x\left(\cos 2 \xi-\frac{U^{T}}{x}\right)^{2}+(S+x)\left(\sin 2 \xi+\frac{V}{S+x}\right)^{2}$

verwandeln lärst. Durch Vergleichung der beiden Ausdrücke erhält man

oder

$$
0=T-x-\frac{U U}{x}-\frac{V V}{S+x}
$$

$\left(\Gamma^{\prime}\right) \ldots x^{3}+(S-T) x^{2}+(U U+V V-S T) x+S U U=0$ $\operatorname{cotg} \alpha \cos \left(\frac{1}{2} \theta^{\prime \prime}-\xi\right)=\cos y \sin \left(\frac{1}{2} \theta^{\prime \prime}-\xi\right)-\operatorname{cotg} l \sin y$

$\operatorname{cotg} \alpha^{\prime} \cos \left(\frac{1}{2} \theta^{\prime \prime}+\xi\right)=\cos y \sin \left(\frac{1}{2} g^{\prime \prime}+\xi\right)-\operatorname{cotg} l \sin y$, woraus sogleich folgt, wenn man $\operatorname{cotg} l=d, \operatorname{cotg} l^{\prime}=d^{\prime}$, $\operatorname{cotg} \alpha=e, \operatorname{cotg} \alpha^{\prime}=o^{\prime}$ setzt:

$\left\{\left(d^{\prime}-d^{\prime}\right) \sin \frac{1}{2} \theta^{\prime \prime} \cos \xi-\left(d^{\prime}+d\right) \cos \frac{1}{2} \theta^{\prime \prime} \sin \xi\right\} \cos y$

$=\left(d^{\prime} \theta-d e^{\prime}\right) \cos \frac{1}{2} \theta^{\prime \prime} \cos \xi+\left(d^{\prime} e+d e^{\prime}\right) \sin \frac{1}{2} \theta^{\prime \prime} \sin \xi$

$\left\{\left(d^{\prime}-d\right) \sin \frac{1}{2} \theta^{\prime \prime} \cos \xi-\left(d^{\prime}+d\right) \cos \frac{1}{2} \theta^{\prime \prime} \sin \xi\right\} \sin \gamma$ $=\frac{1}{2}\left(e-e^{\prime}\right) \sin \theta^{\prime \prime}+\frac{1}{2}\left(e+e^{\prime}\right) \sin 2 \xi$.

Addirt man die Quadrate dieser beiden Gleichungen, und setzt zur Abkürzung:

$$
\begin{aligned}
& \left(d^{\prime}-d\right) \sin \frac{1}{2} \theta^{\prime \prime}=g \cos G ; \quad\left(d^{\prime} e-d e^{\prime}\right) \cos \frac{1}{2} \theta^{\prime \prime}=g \sin G \\
& \left(d^{\prime}+d\right) \cos \frac{1}{2} \theta^{\prime \prime}=h \sin H ; \quad\left(d^{\prime} e+d \theta^{\prime}\right) \sin \frac{1}{2} \theta^{\prime \prime}=h \cos H
\end{aligned}
$$

so erhält man

Soll die Gleichung ( $\mathrm{T}$ ) mögliche Wurzeln haben, so mufs $x$ zwischen 0 und $-S$ fallen, ( $S$ ist positiv); sie hat in der That innerhalb dieser Grenzen wenigstens eine Wurzel, da $\left(\Gamma^{\prime}\right)$ für den Werth von $x=0, s U U$ oder positiv wird, für den Werth $x=-S,-S V V$ oder negativ wird. Setzt man in (Г) $V\left(\frac{x}{-S}\right)=\cos P$, so verwandelt sie sich in:

$$
\cos (2 \xi+P)=\frac{U}{S \cos P}+\frac{V}{S \sin P}
$$

Da $P$ und $2 \xi+P$ jede zwei verschiedene Werthe haben, so kann $\xi$ vier verschiedene Werthe bekommen, wie oben. Es ist jedoch zu bemerken, dafs wenn man $S \sin P$ negativ nimmt man auch zugleich $P$ in dem Winkel $2 \xi+P$ negativ annehmen mufs.

Wenn $\xi$ gefunden ist, findet man leicht die übrigen Stücke des sphärischen Vierecks, und mit diesen die Theile der Pyramide. Schliefslich bemerke ich, dafs ich diese Auflösung des sphärischen Vierecks schon früher in Crelles Journal 7. Bd. p. 105 auf eine astronomische Aufgabe angewandt hahe.

Th. Clausen. 